JASC 12-1-12

\title{
Ultraviolet and visible light detection characteristics of amorphous indium gallium zinc oxide thin film transistor for photodetector applications
}

\author{
Seongpil Chang, Byeong-Kwon Ju ${ }^{\dagger}$ \\ Display and Nanosystem Laboratory, Department of Electrical Engineering, College of Engineering, Korea \\ University, Seoul 136-713, Republic of Korea
}

\begin{abstract}
The ultraviolet and visible light responsive properties of the amorphous indium gallium zinc oxide thin film transistor have been investigated. Amorphous indium gallium zinc oxide (a-IGZO) thin film transistor operate in the enhancement mode with saturation mobility of $6.99 \mathrm{~cm}^{2} / \mathrm{Vs}$, threshold voltage of $13.5 \mathrm{~V}$, subthreshold slope of $1.58 \mathrm{~V} / \mathrm{dec}$ and an on/off current ratio of $2.45 \times 10^{8}$. The transistor was subsequently characterized in respect of visible light and UV illuminations in order to investigate its potential for possible use as a detector. The performance of the transistor is indicates a high-photosensitivity in the off-state with a ratio of photocurrent to dark current of $5.74 \times 10^{2}$. The obtained results reveal that the amorphous indium gallium zinc oxide thin film transistor can be used to fabricate UV photodetector operating in the $366 \mathrm{~nm}$.
\end{abstract}

Key words : a-IGZO TFT, Photoresponse, Photodetector, Oxide-semiconductor

\section{INTRODUCTION}

Transparent oxide semiconductors, such as zinc oxide $(\mathrm{ZnO})$, indium tin oxide (ITO), zinc tin oxide (ZTO), gallium doped zinc oxide (GZO) indium zinc oxide (IZO), and indium gallium zinc oxide (IGZO) have attracted many researchers with their great potential in optoelectronic applications such as flat panel displays, transparent electrodes in solar cells, transparent thin film transistors (TFTs), and flexible transparent TFTs. ${ }^{1-7}$ They have amorphous phase or poly-crystalline phase, oxide semiconductors have higher mobility than a-Si. Especially a-IGZO, has attracted wide attention with its notable advantages over other semiconductors including room-temperature process availability, good-uniformity, high transparency in visible region (400 - $700 \mathrm{~nm}$ ), and high mobility. High mobility of a-IGZO is originated from the larger $n s$-orbital of the metal cation than $2 p$-orbital of oxygen anion. ${ }^{1}$ Moreover a-IGZO

Manuscript received May. 25, 2012;

†Corresponding Author: bkju@korea.ac.kr

Tel: +82-2-3290-3237, Fax: +82-2-3290-3791, Korea Univ.

Dept. of Electrical Engineering, College of Engineering, Korea

Univ., Republic of Korea. thin film transistors also can be used to detect the ultraviolet (UV) which is higher wavelength than $400 \mathrm{~nm}$. In previous arts, Keun Woo Lee et al. reported the photosensitivity of solution processed single walled carbon nanotube blended a-IGZO thin film. This property of a-IGZO thin film is very useful to apply the UV-detector. In present study, we fabricated a-IGZO thin film transistor to investigate the photo-sensing characteristics of the transistor under the illuminations of visible light and UV.

\section{EXPERIMENTAL DETAILS}

Thermally oxidized p-Si $(100, \rho=0.005 \Omega \mathrm{cm})$ with $\mathrm{SiO}_{2}$ of $300 \mathrm{~nm}$ are used as substrates. These substrates are cleaned by acetone, methanol, and de-ionized water in ultra-sonic bath, respectively. We deposited a-IGZO thin film by using radio-frequency (RF) magnetron sputtering. Working pressure and RF-power density are maintained to be about 1.0 $\times 10^{-2}$ Torr and $1.86 \mathrm{~W} / \mathrm{cm}^{2}$ while the a-IGZO of $80 \mathrm{~nm}$ is deposited. Also, sputtering process is carried out at room-temperature. Active layer is patterned by photolithography and lift-off process. As the source-drain (S/D) electrodes, molybdenum (Mo) of $100 \mathrm{~nm}$ is deposited by using direct-current (DC) sputtering at room-temperature. 
S/D electrodes are also patterned by photolithography and lift-off process. Our devices have channel width (W) of 150 $\mu \mathrm{m}$ and channel length (L) of $20 \mu \mathrm{m}$. Finally, to enhance the device performance, devices are annealed at $300^{\circ} \mathrm{C}$ in tube-furnace. Ambient gas and annealing time are nitrogen $\left(\mathrm{N}_{2}\right)$ and 2 hours. Electrical characteristics are measured by semiconductor parameter analyzer (Keithley 4200). In electrical analysis, threshold voltage $\left(\mathrm{V}_{\mathrm{th}}\right)$ is defined by the gate-voltage when induced drain-current of $\mathrm{W} / \mathrm{L} \times 10 \mathrm{nA}$ is induced. ${ }^{9}$ Also, the field effect mobility is estimated by linear fitting the square root of drain-current versus gate-voltage curve at a drain voltage of $10.1 \mathrm{~V}$. The photoresponse properties of the transistor were performed under UV and visible light illuminations by semiconductor parameter analyzer (Keithley 4200) using a white lamp (200 W) and UV lamp with $366 \mathrm{~nm}$.

\section{RESULTS AND DISCUSSION}

Figure 1 shows the drain current-drain voltage characteristics of a-IGZO transistor under various gate voltages. The drain current increases at positive voltages. This indicates that the electrons are generated by the positive gate voltages due to n-type FET characteristics with good gate controllability. The drain current reaches the saturation regime, when the entire channel region is depleted of electrons, i.e, channel is pinched off.

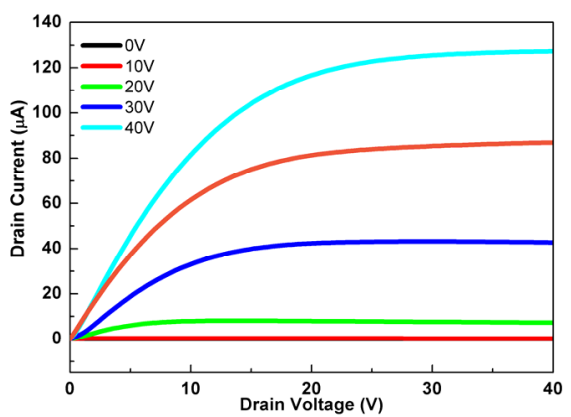

Fig.1. Output characteristics of a-IGZO thin film transistor.

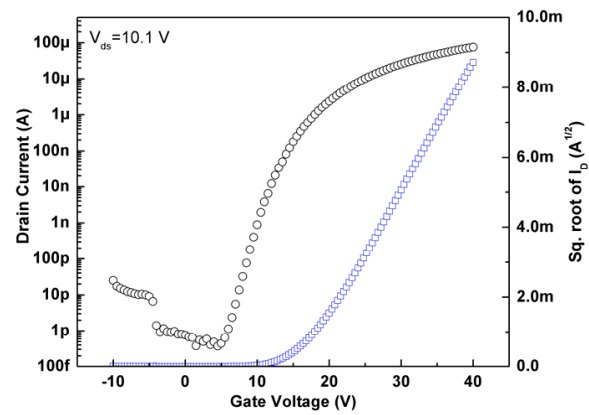

Fig. 2. Transfer characteristics of a-IGZO thin film transistor.
The mobility and threshold voltage parameters of the transistor can be determined by the following relation, ${ }^{10-11}$

$$
I_{d s}=\frac{W}{2 L} \mu_{S A T} C_{i}\left(V_{g}-V_{t h}\right)^{2}
$$

where $\mu_{S A T}$ is the saturation mobility, $C_{i}$ is the capacitance per unit area of the insulator, $I_{d s}$ is the drain-source current, $W$ is the width of channel, $L$ is the channel length, $V_{g}$ is the gate voltage, and $V_{t h}$ is the threshold voltage.

Table 1. Electrical parameters of a-IGZO thin film transistor.

\begin{tabular}{cc}
\hline Parameters & Value \\
\hline Threshold Voltage (V) & 15 \\
Subthreshold Slope (V/dec) & 1.58 \\
On/Off Ratio & $2.45 \times 10^{8}$ \\
Saturation Mobility $\left(\mathrm{cm}^{2} / \mathbf{V s}\right)$ & 7.0 \\
\hline
\end{tabular}

The field effect mobility and threshold voltage values were determined from the plot of drain source current vs. gate voltage under $\mathrm{V}_{\mathrm{ds}}=10.1 \mathrm{~V}$, as shown in Fig. 2. The saturation mobility, threshold voltage, on/off ratio of a-IGZO transistor from $I_{d s}{ }^{1 / 2}-V_{g}$ plot are given in Table 1. The transistor exhibited an on/off ratio of $2.45 \times 10^{8}$ and this value is considerable high. The sub-threshold swing value which is a measure of the turn-on speed of the transistor can be determined by the following relation,

$$
S=\left[\frac{d\left(\log I_{d s}\right)}{d V_{g}}\right]^{-1}
$$

The $\mathrm{S}$ value for the transistor was determined from Fig. 2 and was given in Table 1 . The $\mathrm{S}$ value indicates the presence of trap behavior and interface quality between the dielectric and a-IGZO active layer. The interface trap density for the transistor can be calculated by the following relation: ${ }^{13-14}$

$$
D_{i t}=\left[\frac{S \log (e)}{k T / q}-1\right] \frac{C_{i}}{q}
$$

where $\mathrm{C}_{\mathrm{i}}\left(11.5 \mathrm{nF} / \mathrm{cm}^{2}\right.$ for $\left.\mathrm{SiO}_{2}\right)$ is the capacitance of dielectric layer, $k$ is the Boltzmann constant, $\mathrm{q}$ is the electronic charge and $\mathrm{T}$ is the temperature. The $\mathrm{D}_{\text {it }}$ value for the transistor was found to be $1.83 \times 10^{12} \mathrm{~cm}^{-2} \mathrm{eV}^{-1}$. We have evaluated that this $\mathrm{D}_{\mathrm{it}}$ value is considerable higher.

This high $\mathrm{D}_{\text {it }}$ value makes a contribution to the capacitance of the dielectric layer and in turn, the mobility of the transistor increases. Ultraviolet and visible light detection characteristics of a-IGZO thin film transistor were investigated under white light and UV illuminations. Figure 3 shows the $\mathrm{I}_{\mathrm{ds}}-\mathrm{V}_{\mathrm{ds}}$ curves obtained white light and UV illumination $\left(366 \mathrm{~nm}\right.$ ) with $\mathrm{V}_{\mathrm{g}}=30 \mathrm{~V}$. The drain current under these illuminations increases due to photogeneration of electron-hole pairs in the active layer of the transistor. 


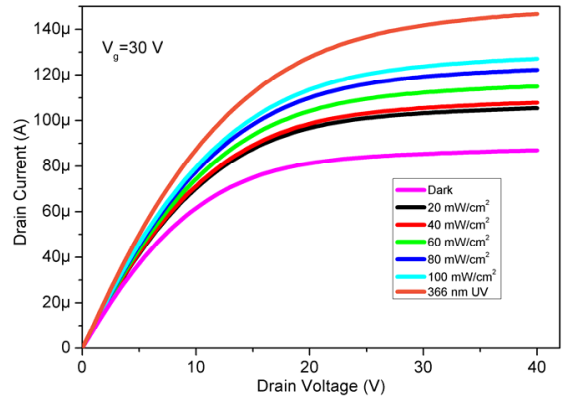

Fig. 3. Output characteristics of a-IGZO thin film transistor under white light and UV illuminations

The effect of UV illumination is higher than that of white light illumination. The UV illumination increases strongly the drain current, because the photon energy of UV illumination at $366 \mathrm{~nm}$ is higher than the a-IGZO optical band gap.

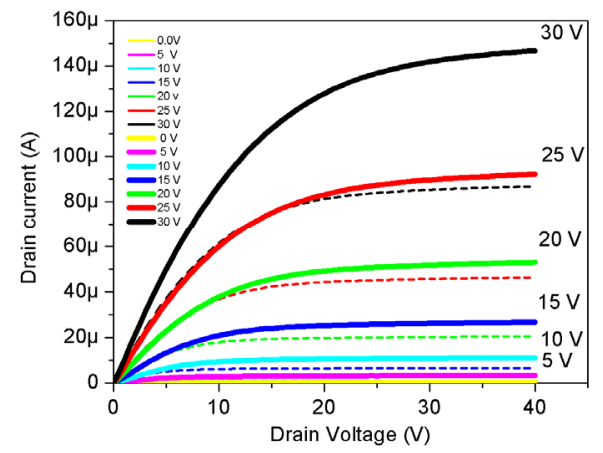

Fig. 4. Output characteristics of a-IGZO thin film transistor under UV illumination of $366 \mathrm{~nm}$

The photoresponse of the transistor under visible light can be analyzed by the following relation, ${ }^{15}$

$$
R_{L / D}=\frac{I_{\text {photo }}}{I_{\text {dark }}}=A P^{\gamma}
$$

where $\mathrm{I}_{\text {photo }}$ is the drain source current under illumination, $\mathrm{I}_{\text {dark }}$ is the drain source current under dark, $A$ is a constant and $P$ is the illumination intensity and $\gamma$ is a constant. The plot of $\mathrm{R}_{\mathrm{L} / \mathrm{D}}$ vs illumination intensity for a-IGZO thin film transistor was plotted via Fig. 3 and in the ON-state, it was seen that the drain current increases linearly with illumination intensity. The $\gamma$ value for the transistor was found to be 0.12 . This value for the a-IGZO transistor indicates the participation of a recombination path.

For analysis of UV detection of the transistor, the output characteristics of a-IGZO thin film transistor under UV illumination of $366 \mathrm{~nm}$ were measured and are given in Fig. 4. As seen in Fig.4, the UV illumination increases the drain current for various gate voltages. In order to analyze the photovoltaic effect and photoconductivity of the transistor, we measured drain current under dark and UV illumination for various gate voltages, because when the transistor is illuminated, photovoltaic and photoconductivity effects may occur in the active layer of the transistor. When the transistor is in the ON-state the photocurrent is dominated by the photovoltaic effect. Whereas, in the off state, photoconductivity effect is significant for the transistor. As seen in Fig.5, the a-IGZO transistor follows both the photovoltaic effect in the turn-on state and the photoconductive effect in the turn-off state.

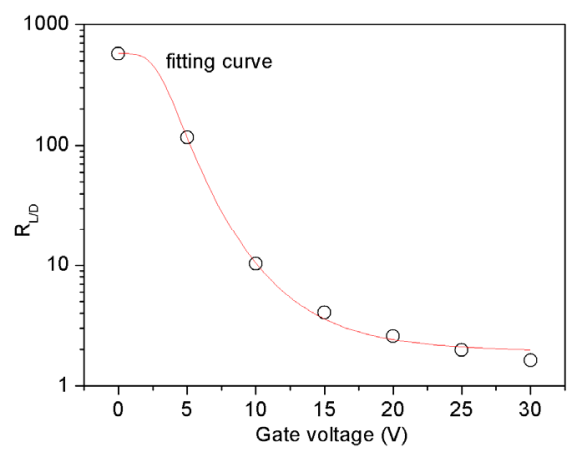

Fig. 5 Plot of photoresponse versus gate voltage for UV illumination of $366 \mathrm{~nm}$

In order to analyze photovoltaic or photoconductivity effects on photoresponse properties of the transistor, we plotted the photoresponse as a function of gate voltage. As seen in Fig.5, the $\mathrm{R}_{\mathrm{L} / \mathrm{D}}$ value for the transistor was obtained at the switch-off voltage. But, the lowest $\mathrm{R}_{\mathrm{L} / \mathrm{D}}$ occurs at $30 \mathrm{~V}$ gate bias. We have evaluated that the photogeneration is maximum at zero gate bias, at off-state of the transistor; the carrier injection and thermal generation have the smallest contribution to photogeneration and in turn photogenerated current is have highest contribution. Whereas, the photogeneration is minimum at $30 \mathrm{~V}$ gate bias, because at this bias, the dark current also increases. The obtained results show that the photoresponse properties of a-IGZO transistor are modulated by gate bias. The photoresponse of a-IGZO transistor was analyzed and the following function was obtained as

$$
R_{L / D}=A_{2}+\left(A_{1}-A_{2}\right) \cdot\left(1+\left(V_{g} / V_{g o}\right)^{-p}\right.
$$

where $\mathrm{A}_{1}, \mathrm{~A}_{2}, \mathrm{~V}_{\mathrm{go}}$ and $\mathrm{p}$ are constants. For the transistor, the constants in Eq.5 were found to be $A_{1}=574.14847$, $\mathrm{A}_{2}=1.88469, \mathrm{~V}_{\mathrm{go}}=3.54488$ and $\mathrm{p}=4.03234$. We have evaluated that the transistor shows a high photoresponse at off-state of $5.74 \times 10^{2}$. This suggests that the IGZO transistor can find use in two-terminal photodetector applications. 


\section{CONCLUSION}

The photoresponse properties of the IGZO transistor was investigated under visible light and UV illuminations. The electrical parameters like mobility, threshold voltage, photoresponse, voltage swing, and ON/OFF current ratio were extracted from experimental data. The studied thin film transistor is expected to find application as UV photodetector in the UV region at $366 \mathrm{~nm}$.

\section{ACKNOWLEDGMENT}

This research was supported by Basic Science Research Program through the National Research Foundation of Korea(NRF) funded by the Ministry of Education, Science and Technology (No.2009-0083126), the Industrial strategic technology development program (KI002182, TFT backplane technology for next generation display) funded by the Ministry of Knowledge Economy(MKE, Korea), and supported by a Korea University Grant.

\section{REFERENCES}

[1] Nomura, H. Ohta, A. Takagi, T. Kamiya, M. Hirano, and H. Hosono, Nature (London) 432, 488 (2004).

[2] J. -W. Kim, H. -S. Kang, and S. Y. Lee, J. Elec. Eng. \& Tech. 1, 98 (2006).

[3] R. L. Hoffman, B. J. Norris, and J. F. Wager, Appl. Phys. Lett. 82, 733 (2003).

[4] J. -H. Kwon, J. -H. Seo, S. -I. Shin, and B. -K. Ju, J. Phys. D: Appl. Phys. 42, 065105 (2009).

[5] H. Q. Chiang, J. F. Wager, R. L. Hoffman, J. Jeong, and D. A. Keszler, Appl. Phys. Lett. 86, 013503 (2005).

[6] Dhananjay, and C, W. Chu, Appl. Phys. Lett. 91, 132111 (2007).

[7] S. Chang, Y. -W. Song, S. Lee, S. Y. Lee, and B. -K. Ju, Appl. Phys. Lett. 92, 192104 (2008).

[8] K. W. Lee, K. Y. Heo, and H. J. Kim, Appl. Phys. Lett. 94, 102112 (2009).

[9] J. -S. Park, J. K. Jeong, Y. -G. Mo, H. D. Kim, and C. -J. Kim, Appl. Phys. Lett. 93, 033513 (2008).

[10] P. Servati, A. Nathan, and G. A. J. Amaratunga, Phys. Rev. B 74, 245210 (2006).

[11] A. Tsusima, H. Koezuka, T. Ando, Appl. Phys. Lett. 49,1210 (1986).

[12] T.-C. Fund, C.-S. Chuang, K. Nomura, H.-P. D. Shieh, H. Hosono, J. Kanicki, Journal of Information Display 9, 21 (2008).

[13] G. Horowitz, Adv. Funct. Mater. 13, 53 (2003).

[14] R.N. Christopher, C.D. Frisbie, D.A. da Silva Filho, J.L. Bredas, C.E. Paul, R.M. Kent Chem. Mater. 164436 (2004).

[15] J.D. Gallezot, S. Martin, and J. Kanicki, in Proc. Int. Display Research Conf. (IDRC), pp. 407-410 (2001).

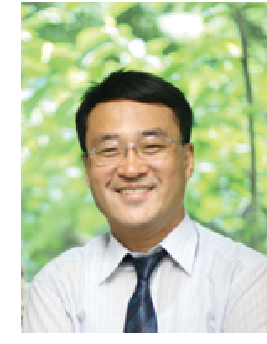

Byeong-Kwon Ju was born in Jechon, Republic of Korea, on 1962. He received the Ph.D. in semiconductor engineering from Korea University in 1995. From 1988, he joined the KoreaInstitute of Science and Technology(KIST), Seoul, where he was engaged in development of mainly flat panel display and MEMS technology as a principal research scientist. In 1996, he spent 6months as a visiting research fellow at Microelectronics Centre, University of South Australia, Australia. Since 2005, he has been a professor of Dept. EE of Korea Universitywith his main interest in flexible electronics (OLED, OTFT), field emission devices, Si-micromachining and carbon nanotube-based nano systems. And also he is a programdirector of National Research Lab. - Display and Nanosystem Lab. supported by Korean government and Head of SMD OLED Center of Korea University. Prof. $\mathrm{Ju}$ is a member ofSociety for Information Display (SID), The Korean Information Display Society(KIDS), The Institute of Electronics Engineers of Korea(IEEK) and The Korean Institute of Electrical Engineers(KIEE).

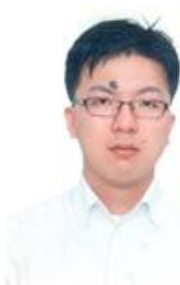

Seongpil Chang was born in Ansan, Kyunggi-do, Republic of Korea. He received B.S. the degree in electronic and information engineering from Seoul national University of Technology, Seoul, Korea, in 2007, and the M.S. degree in electrical engineering from Korea University, Seoul, Korea, in 2009. From 2009, he is currently working toward the $\mathrm{Ph}$. D. degree in electrical engineering at Korea University, Seoul, Korea. In 2007, he joined Korea Institute of Science and Technology (KIST), Seoul, as a Student Researcher, and has researched for the oxide-semiconductor and their applications. His current research interests include oxide semiconductors for flexible electronics and solid-state electronics. 